\author{
Adrian Spreng \\ Peter Netzer \\ Joerg Mattich \\ Hans-Peter Dinkel \\ Peter Vock \\ Hanno Hoppe
}

\section{Importance of extracolonic findings at IV contrast medium-enhanced CT colonography versus those at non-enhanced CT colonography}

Received: 11 February 2005

Revised: 24 April 2005

Accepted: 29 April 2005

Published online: 18 June 2005

(C) Springer-Verlag 2005

\begin{abstract}
To compare the clinical importance of extracolonic findings at intravenous (IV) contrast-enhanced CT colonography versus those at nonenhanced CT colonography. IV contrast medium-enhanced $(n=72)$ and non-enhanced $(n=30)$ multidetector $\mathrm{CT}$ colonography was performed in 102 symptomatic patients followed by conventional colonoscopy on the same day. The impact of extracolonic findings on further work up and treatment was assessed by a review of patient records. Extracolonic findings were divided into two groups: either leading to further work up respectively having an impact on therapy or not. A total of 303 extracolonic findings were detected. Of those, $71 \%$ $(215 / 303)$ were found on IV contrastenhanced CT, and 29\% (88/303) were found on non-enhanced CT colonography. The extracolonic findings in
\end{abstract}

$25 \%(26 / 102)$ of all patients led to further work up or had an impact on therapy. Twenty-two of these patients underwent $\mathrm{CT}$ colonography with IV contrast enhancement, and four without. The percentage of extracolonic findings leading to further work up or having an impact on therapy was higher for IV contrast-enhanced $(31 \%$; $22 / 72)$ than for non-enhanced $(13 \%$; $4 / 30)$ CT scans $(P=0.12)$. IV contrastenhanced CT colonography produced more extracolonic findings than non-enhanced CT colonography. A substantially greater proportion of findings on IV contrast-enhanced CT colonography led to further work up and treatment than did nonenhanced CT colonography.

Keywords Extracolonic findings . Virtual colonoscopy $\cdot$ CT colonography $\cdot$ Abdominal CT

\section{Introduction}

First described by Vining et al. in 1994, CT colonography, or so-called "virtual colonoscopy," is a rapidly evolving minimally invasive technique for examination of the entire colon and detection of colorectal neoplasms [1]. Previous studies have shown that CT colonography has the potential to become a valuable clinical screening method for colorectal neoplasms [2-6].

In contrast to other screening tools for colorectal cancer, such as conventional colonoscopy and barium enema, CT colonography allows for simultaneous visualization of extracolonic pathologies within the abdominal organs, ves- sels, bone, and soft tissue [7]. Such findings are reported to be useful in staging cancer, explaining a patient's presenting symptoms, and detecting other potentially serious disorders [8]. On the other hand, extracolonic findings may involve phenomena already known or may lead to unnecessary further work up, which can be problematic for both ethical and economical reasons $[9,10]$.

Only a few studies have examined the clinical relevance of extracolonic findings at CT colonography [7, 9-12]. These studies examining non-enhanced CT scans of the abdomen report large differences in the prevalence of extracolonic findings and the need for additional work up. To our knowledge, the present study is the first to compare the 
clinical relevance of extracolonic findings at IV contrastenhanced CT colonography with those at non-enhanced CT colonography.

\section{Patients and methods}

\section{Patients}

One hundred and two adult patients referred to our gastroenterology clinic for conventional colonoscopic evaluation of symptoms (including hematochezia, positive hemoccult test, iron deficiency anemia, or personal or family history of colonic neoplasms) were enrolled in the study. The study's trial protocol was approved by the institute's ethics committee and performed in accordance with the revised Declaration of Helsinki of 1989. Each patient provided written consent after being fully informed regarding the study protocol. In all patients, CT colonography was followed by conventional colonoscopy the same morning.

\section{CT colonography}

On the day before the examinations, each patient was given a wet bowel preparation consisting of 41 of methylcellulose as prescribed by the participating gastroenterologist. No oral contrast medium was administered. CT colonography was performed with an Asteion four-channel multidetector CT scanner (Toshiba, Tokyo, Japan). A flexible rubber catheter with a rectal balloon was inserted into the rectum by the investigating radiologist. The patient's colon was then insufflated with room air according to the patient's tolerance. The catheter was clamped and left in the rectum, and a single supine scout CT image was obtained to verify adequate bowel distension. If bowel distension was inadequate, additional air was insufflated into the rectum. Once bowel distension was adequate, CT colonography was performed. In 72 of the 102 patients, $120 \mathrm{ml}$ (flow-rate: $3 \mathrm{ml} / \mathrm{s}$; scan delay: $60 \mathrm{~s}$ ) of iopromide IV contrast medium containing $300 \mathrm{mg} / \mathrm{ml}$ iodine (Ultravist 300, Berlex Laboratories, Montville, USA) was power injected, followed first by $\mathrm{CT}$ colonography in the supine position in a craniocaudal direction to image the entire region of the colon and rectum, then by examination in the prone position without additional injection of contrast medium. In 30 of the 102 patients, administration of IV contrast medium was contraindicated due to an elevated creatinine level, known renal insufficiency, or contrast media allergy.

CT parameters included $4 \times 2-\mathrm{mm}$ detector collimation, $120 \mathrm{kV}, 0.75$-s gantry rotation, $200 \mathrm{mAs}$ and a pitch of 1.375. The entire abdomen and pelvis were scanned during a breath hold of approximately $30 \mathrm{~s}$. Axial CT images were reconstructed as $2-\mathrm{mm}$ slices with a $1-\mathrm{mm}$ reconstruction interval.

Image analysis

Methods for polyp detection have been described elsewhere [6]. The reconstructed supine and prone datasets were transferred to an Advantage Windows workstation (Version 4.0, General Electric Medical Systems, Milwaukee, WI) running on Sun Ultra Sparc 60 hardware (Sun Microsystems, Mountain View, CA) featuring two Sun Ultra Sparc II $450 \mathrm{MHz}$ central processing units and 2 gigabytes of random access memory. Images for extracolonic findings were read by a board-certified radiologist and a radiology resident. Since the images were read during clinical routine they were not always evaluated by the same readers throughout the study. The supine acquisitions were IV contrast enhanced in 72 patients and non-enhanced in 30 patients; no further IV contrast medium was administered for prone scans. Images were viewed as continuous 2$\mathrm{mm}$ axial sections with regular abdominal contrast window display settings (level $50 \mathrm{H}$, width $450 \mathrm{H}$ ). The window/ level settings could be adjusted manually at the workstation. The basal portions of the lungs were also viewed using standard lung window settings (level $-450 \mathrm{H}$, width $1,850 \mathrm{H}$ ). If necessary, additional multiplanar reformatting was performed using the workstation. Both readers were unaware of the patients' medical history, but knew that the examined patient cohort consisted of symptomatic patients. One radiologist per study was responsible for dictating an official CT colonography report, which included both intra- and extracolonic findings plus suggestions for possible work-up procedures. The report was sent to the referring clinician.

\section{Definitions}

Extracolonic findings were divided into two groups: either leading to further work-up respectively having an impact on therapy or not. Extracolonic findings were classified by organ system as hepatic (lesion, fatty liver), vascular (aneurysm, thrombosis, sclerosis, varices), gallbladder (cholecystitis, polyps) or cholangio (cholestasis), pulmonary (nodule, infiltrate, emphysema, fibrosis) or pleural (pleuritis, effusion, calcification, thickening), musculoskeletal(mass including hematoma or lipoma, osteolysis, arthrosis), urogenital (renal mass, renal cyst, renal duplication, nephrolithiasis, adrenal mass, prostatic enlargement, testicular hydrocele, uterine myoma, any calcification), cardiac (pericardial effusion, coronary artery calcification, cardiomegaly), splenic (lesion, splenomegaly, accessory spleen), pancreatic (mass, 
Table 1 The number of extracolonic findings at CT colonography in 102 patients, number of previously known findings, and corresponding numbers of findings on CT scans with and without IV contrast enhancement

A patient could have more than one finding

\begin{tabular}{|c|c|c|c|c|c|}
\hline \multirow[t]{2}{*}{ Finding } & \multirow{2}{*}{$\begin{array}{l}\text { No. of } \\
\text { findings }\end{array}$} & \multicolumn{2}{|c|}{ No. of previously known findings } & \multirow{2}{*}{$\begin{array}{l}\text { Findings on IV } \\
\text { contrast-enhanced } \\
\text { CT scans }\end{array}$} & \multirow{2}{*}{$\begin{array}{l}\text { Findings on } \\
\text { non-enhanced } \\
\text { CT scans }\end{array}$} \\
\hline & & IV contrast & Non-enhanced & & \\
\hline Hepatic & 40 & 3 & 2 & 30 & 10 \\
\hline Vascular & 48 & 6 & 0 & 33 & 15 \\
\hline Gallbladder/cholangio & 18 & 0 & 0 & 13 & 5 \\
\hline Pulmonary/pleural & 32 & 3 & 0 & 24 & 8 \\
\hline Musculoskeletal & 13 & 1 & 0 & 9 & 4 \\
\hline Urogenital & 65 & 2 & 0 & 48 & 17 \\
\hline Cardiac & 15 & 0 & 0 & 7 & 8 \\
\hline Splenic & 8 & 0 & 0 & 6 & 2 \\
\hline Pancreatic & 3 & 1 & 0 & 2 & 1 \\
\hline Hernia & 20 & 1 & 0 & 14 & 6 \\
\hline Other & 41 & 2 & 0 & 29 & 12 \\
\hline Total no. of findings & 303 & 19 & 2 & 215 & 88 \\
\hline
\end{tabular}

pancreatitis, calcification), hernia (inguinalhernia, hiatal hernia), or other (enlarged lymph nodes, ascites, esophageal or gastric mass). The definition of a fatty liver on non-contrast-enhanced CT was mean CT Hounsfield units lower in the liver than in the spleen or on contrast-enhanced CT if the liver is less attenuating than muscle, since comparison with the spleen is not accurate on contrast-enhanced CT scans [13]. Prostate enlargement was defined as a prostate volume larger than $25 \mathrm{ml}$. Enlarged abdominal lymph nodes were defined as short axis larger than $10 \mathrm{~mm}$.

\section{Follow-up}

The minimum follow-up time after CT colonography was 6 months, the maximum 30 months. The files for each patient with extracolonic findings were reviewed to determine the number and results of any examination [e.g., CT, ultrasound (US), angiography, biopsy, ERCP, MRI, gastroscopy) performed because of extracolonic findings at CT colonography. The numbers and results of such examinations or surgical procedures performed on the basis of CT colonography were tabulated. An effort was made to determine whether any extracolonic findings were known before or if they were initially discovered at CT colonography and whether the extracolonic finding was made at IV contrastenhanced or non-enhanced CT colonography.

\section{Statistics}

The chi-squared test with Yates' continuity correction was performed to compare the distribution of extracolonic findings made with and without IV contrast enhancement. The age and sex distribution of patients with unenhanced and IV contrast-enhanced CT-colonography was calculated and tested for degrees of difference using the Student's $t$-test or chi-squared test, respectively. A $P$-value of less than 0.05 was considered significant.

\section{Results}

One hundred and two subjects were enrolled in the study (63 men, 39 women; age range: 20-91 years; mean age: 66 years). Complete conventional colonoscopy to the cecum was achieved in 94 patients. No patient had to be excluded from evaluation of extracolonic findings. In 72 patients CT colonography was performed after application of IV contrast medium; in 30 patients IV contrast medium was contraindicated. Of the 72 patients receiving IV contrast, 46 were male and 26 were female, and of the 30 patients not receiving IV contrast 17 were male and 13 were female

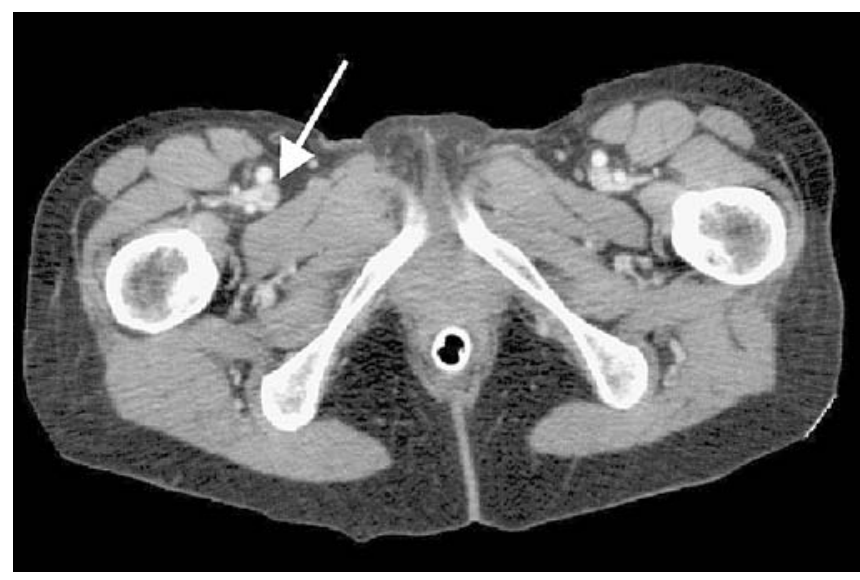

Fig. 1 IV contrast-enhanced CT scan of a 66-year-old female patient with known metastasis of an ovarian carcinoma to the right iliac muscle shows previously unknown thrombosis of the right femoral vein (arrow), probably due to compression by the muscle tumor. Successful treatment with heparin 
Table 2 Extracolonic findings at IV contrast enhanced CT colonography that resulted in additional work-up

\begin{tabular}{|c|c|c|c|c|}
\hline \multicolumn{5}{|c|}{ IV contrast enhanced extracolonic findings resulting in additional work up } \\
\hline Finding & $\begin{array}{l}\text { No. resulting } \\
\text { in work up/no. } \\
\text { of findings }\end{array}$ & Type of work up & Results of work up & $\begin{array}{l}\text { No. } \\
\text { resulting } \\
\text { in surgery }\end{array}$ \\
\hline $\begin{array}{l}\text { Abdominal aortic } \\
\text { aneurysm }(>4 \mathrm{~cm})\end{array}$ & 4 & $\begin{array}{l}\text { CT }(n=2), \text { CT follow-up }(n=1), \\
\text { angiography }(n=1)\end{array}$ & $\begin{array}{l}\text { Abdominal aortic aneurysm }(n=4) \\
\text { with stenting }(n=1) \text { or aortoduodenal } \\
\text { fistula }(n=1)\end{array}$ & 2 \\
\hline Pancreatic mass & 2 & Surgery $(n=2)$ & $\begin{array}{l}\text { Pancreatitis }(n=1), \text { anastomosis } \\
\text { insufficiency }(n=1)\end{array}$ & 2 \\
\hline Hepatic lesion & 1 & CT guided biopsy & Cholangio-Ca & 1 \\
\hline Pelvic mass & 2 & Laparoscopy $(n=2)$ & $\begin{array}{l}\text { Ovarian abscess }(n=1) \\
\text { Abscess caused by sigma } \\
\text { diverticulitis }(n=1)\end{array}$ & 2 \\
\hline Adrenal mass & 1 & $\mathrm{CT}$ & Adenoma & 0 \\
\hline Cholecystitis & 2 & US $(n=2)$ & $\begin{array}{l}\text { Cholecystitis with antibiotic } \\
\text { treatment }(n=2)\end{array}$ & 1 \\
\hline Esophageal mass & 1 & Gastroscopy & Tumor & 1 \\
\hline Lung nodule & 1 & $\mathrm{CT}$ and PET & Lung cancer & 1 \\
\hline Musculoskeletal mass & 1 & MRI & Carcinoma & 1 \\
\hline Renal mass & 1 & Staging CT & Renal cell cancer & 1 \\
\hline Iliacal artery aneurysm & 1 & Angiography & Aneurysm with stenting & 1 \\
\hline Cholestasis & 1 & $\begin{array}{l}\text { ERCP, liver biopsy, } \\
1 \text { aboratory tests }\end{array}$ & $\begin{array}{l}\text { Liver cirrhosis, abnormal } \\
\text { liver function tests }\end{array}$ & 0 \\
\hline Thrombosis & 3 & $\begin{array}{l}\text { Heparinization based on } \\
\text { initial findings }(n=3)\end{array}$ & Regression of thrombosis $(n=3)$ & 0 \\
\hline Pleuritis & 1 & $\mathrm{CT}$ & Pleural effusion & 0 \\
\hline
\end{tabular}

$(P=0.6)$. For the 72 patients receiving IV contrast, the mean age was 64.7 for males and 69.8 for females $(P=0.1)$, and for the patients not receiving IV contrast 65.8 for males and 65.1 for females $(P=0.9)$.

Conventional colonoscopy found a high number of colorectal neoplasms, which was 122 lesions (carcinoma, $n=$ 8; adenoma, $n=67$; hyperplastic bowel mucosa, $n=47$ ) in 49 patients. The results of polyp detection at CT colonography were reported earlier [6].

A total of 303 extracolonic findings were recorded, of which $71 \%(215 / 303)$ were seen at IV-enhanced CT colo- nography and $29 \%(88 / 303)$ at CT colonography without enhancement (Table 1). Eighty nine percent (91/102) of patients had at least one extracolonic finding (Fig. 1). Of these patients, $75 \%$ (68/91) underwent CT scan with IV contrast enhancement, and 25\% (23/91) without. Eleven percent of patients $(11 / 102)$ had no extracolonic findings. Of these, 4\% (4/102) underwent CT scan with IV contrast enhancement and 7\% (7/102) without. Ninety-four percent (68/72) of patients with IV contrast-enhanced CT colonography had extracolonic findings, compared to $77 \%$ (23/30) without IV contrast enhancement $(P=0.02)$.
Table 3 Extracolonic findings at non-enhanced CT colonography that resulted in additional work up

\begin{tabular}{|c|c|c|c|c|}
\hline \multicolumn{5}{|c|}{ Non-enhanced CT colonography findings resulting in additional work up } \\
\hline Finding & $\begin{array}{l}\text { No. resulting } \\
\text { in work up/no. } \\
\text { of findings }\end{array}$ & $\begin{array}{l}\text { Type of } \\
\text { work up }\end{array}$ & $\begin{array}{l}\text { Results of } \\
\text { work up }\end{array}$ & $\begin{array}{l}\text { No. resulting } \\
\text { in surgery }\end{array}$ \\
\hline Hepatic lesion & 1 & CT & Metastasis & 1 \\
\hline Ovarian mass & 1 & Staging CT & $\begin{array}{l}\text { Ovarian cancer, } \\
\text { no metastasis }\end{array}$ & 1 \\
\hline Adrenal mass & 1 & MRI & Adenoma $(1 \mathrm{~cm}, 5 \mathrm{HU})$ & 0 \\
\hline Pulmonary fibrosis & 1 & $\begin{array}{l}\text { High resolution } \\
\text { chest } \mathrm{CT}\end{array}$ & Pulmonary fibrosis & 0 \\
\hline
\end{tabular}




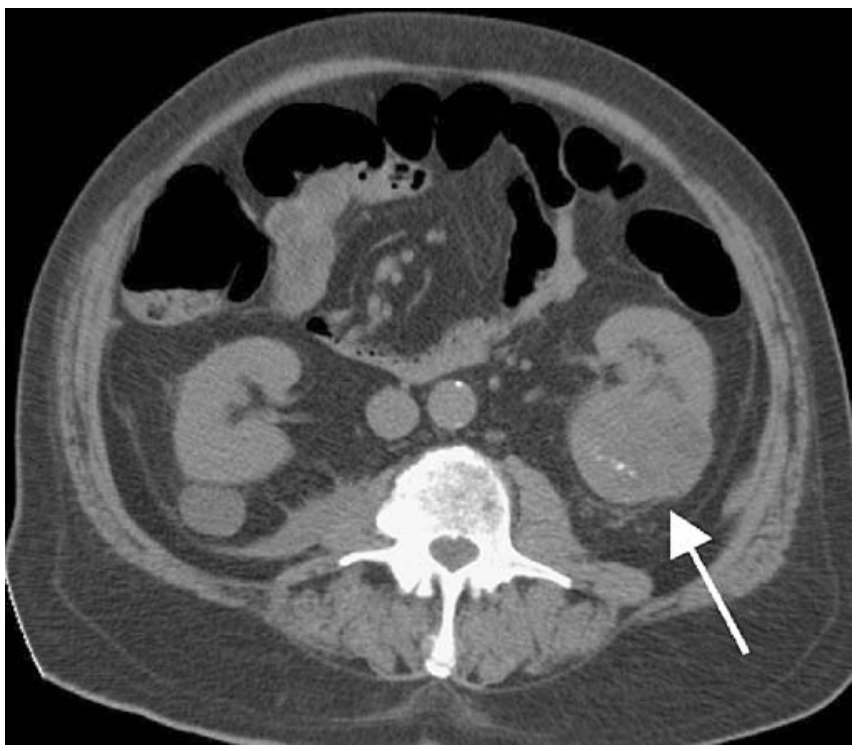

Fig. 2 Non-enhanced CT colonography of an 80-year-old male patient with partially calcified, mainly solid renal mass of the left kidney (arrow). Further work up was precluded by death of patient soon afterwards of unrelated cause

Seven percent (21/303) of extracolonic findings were previously known, including hepatic lesions $(n=5)$, vascular aneurysms $(n=6)$, lung nodules $(n=3)$, an adrenal mass, ovarian cyst, inguinal hernia with bowel, metastasis of the iliac muscle, esophageal mass, pancreatic metastasis, and gastric carcinoma.

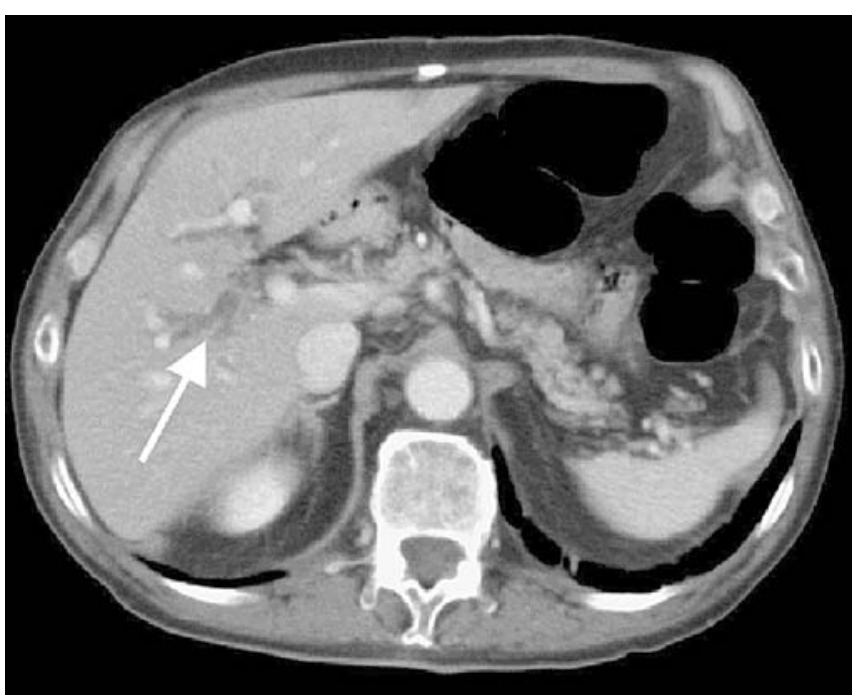

Fig. 3 IV contrast-enhanced CT colonography of a 59-year-old male patient with a surgically treated esophageal carcinoma. Dilatation of the bile ducts (arrow) was initially found on CT colonography. Subsequently, an ERCP was performed, which showed dilated intrahepatic bile ducts, but ruled out biliary calculi or tumor. Finally liver biopsy confirmed the diagnosis of liver cirrhosis
The extracolonic findings in $25 \%$ (26/102) of all patients led to further work up or had an impact on therapy. Twentytwo of these patients had a CT colonography with IV contrast enhancement (Table 2), and four without (Table 3). Calculated per patient, $31 \%(22 / 72)$ of all patients with IV contrast enhancement and 13\% (4/30) of all patients without underwent further work up $(P=0.12)$. Of these, $32 \%$ $(22 / 68)$ of patients with IV contrast and $17 \%(4 / 23)$ of patients without IV contrast had further work up $(P=0.26)$.

One patient died before work up could be performed (Fig. 2). Nineteen patients underwent additional imaging examinations following CT colonography, including CT ( $n=$ $10)$, CT-guided biopsy $(n=1)$, US ( $n=2)$, angiography
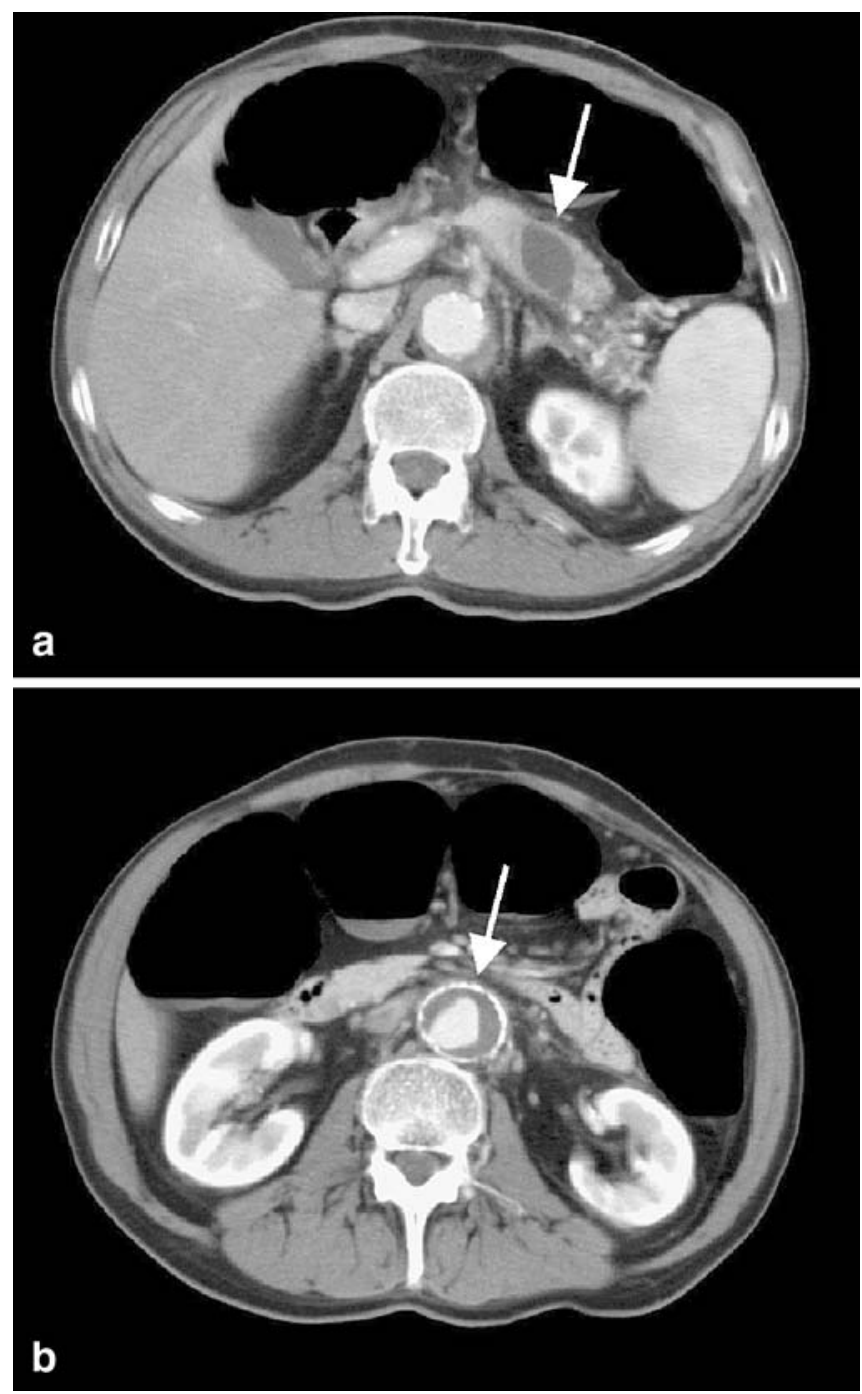

Fig. 4 a, b CT colonography with IV contrast enhancement of a 66year-old male patient with a previously unknown, well-delineated $3.3 \times 2-\mathrm{cm}$ cystic pancreatic mass (a, arrow). No other abdominal masses or suspected metastatic disease was present. The patient underwent surgery that revealed a pancreatic pseudocyst with chronic atrophic and sclerosing pancreatitis. b In addition an abdominal aortic aneurysm was found (arrow) 


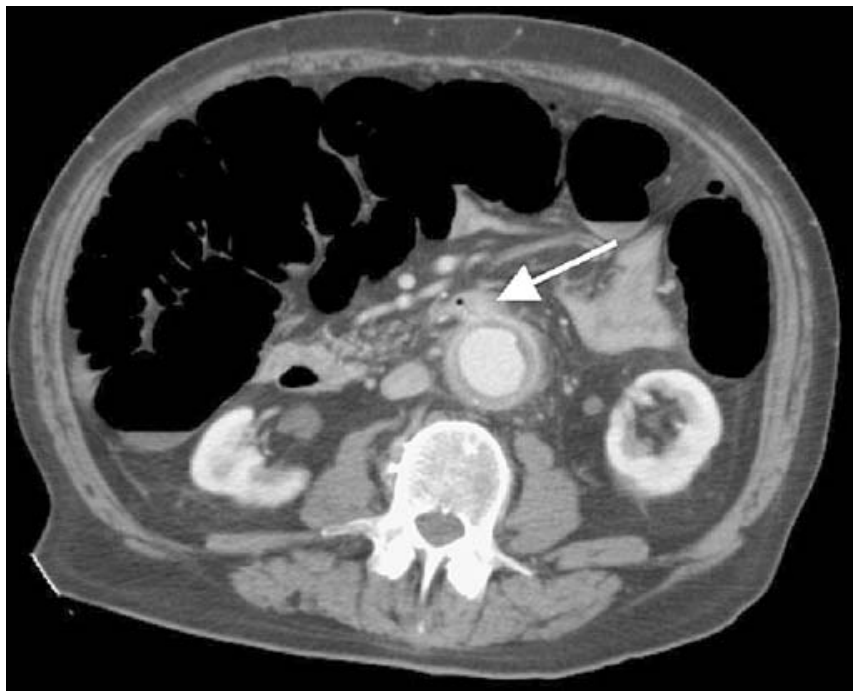

Fig. 5 Inflamed abdominal aortic aneurysm with an aortoduodenal fistula (arrow) on IV contrast-enhanced CT colonography in an 81year-old male patient. The aneurysm was successfully treated in an emergency operation

$(n=2)$, MRI ( $n=2)$, PET $(n=1)$, endoscopic retrograde cholangiopancreatography $(n=1)$ (Fig. 3), and gastroscopy $(n=1)$. Fifteen patients underwent surgery, including abdominal aortic aneurysm repair $(n=1)$, pancreas resection $(n=1)$ (Fig. 4), resection of an aortoduodenal fistula $(n=1)$ (Fig. 5), duodenal anastomosis insufficiency repair $(n=1)$, liver metastasis/tumor resection $(n=2)$, ovarian cancer resection $(n=1)$, pelvic abscess drainage $(n=2)$, gallbladder resection $(n=1)$, esophageal tumor resection $(n=1)$, lung

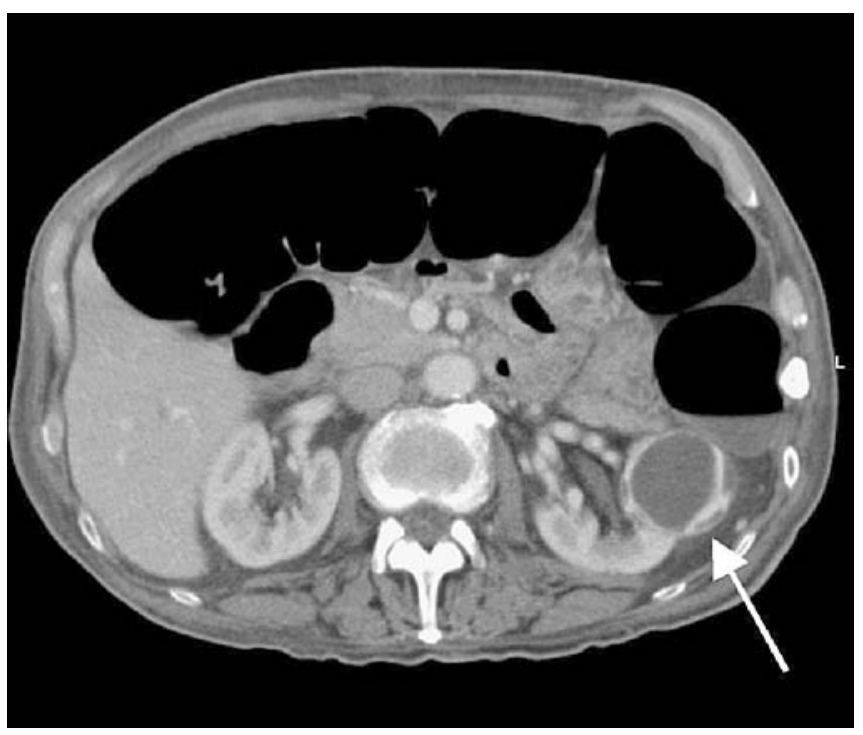

Fig. 6 Complex cystic mass with thickened septations of the left kidney (arrow) in a 76-year-old male patient (IV contrast-enhanced CT scan). After radiological staging (negative) the patient underwent successful nephrectomy. The specimen exhibited a renal cell carcinoma cancer resection $(n=1)$, musculoskeletal tumor resection $(n=1)$, renal cell carcinoma resection $(n=1)$ (Fig. 6), and iliacal artery aneurysm repair $(n=1)$. A follow-up CT was performed after more than 6 months in one case of abdominal aortic aneurysm. Follow-up CT was also performed in three patients with known esophageal mass, known pancreatic metastasis, and known gastric carcinoma, which would have been performed unrelated to extracolonic findings on CT colonography. In one patient with suspected pleuritis a chest CT scan was performed for work-up. Another patient received a high resolution chest CT scan for evaluation of suspected pulmonary fibrosis. One staging CT was performed in a patient with ovarian cancer. Follow-up revealed no important lesions missed at CT colonography.

Additionally to the performed work-up shown in Table 2, the following suggestions for possible work-up procedures were given: Ultrasound was recommended in eight patients (non-enhanced CT, $n=4$; IV contrast-enhanced CT, $n=4$ ), CT in three patients (non-enhanced CT, $n=1$; IV contrast-enhanced CT, $n=2$ ), and MRI in two patients (non-enhanced CT, $n=2$ ).

\section{Discussion}

The primary purpose of the present study was to evaluate the impact of IV contrast medium application on the detection of extracolonic findings and further patient work up in symptomatic patients referred for conventional colonoscopy. The results show that IV contrast-enhanced CT colonography produced more extracolonic findings than non-enhanced CT colonography. Moreover, the percentage of extracolonic findings leading to further work up or having an impact on therapy was higher for IV contrastenhanced than for non-enhanced CT scans, which can be explained by the fact that an IV contrast-enhanced abdominal CT scan may produce a higher number of extracolonic findings than a non-enhanced abdominal CT scan (e.g., visualization of organ lesions without mass effect or vascular thrombosis).

In the present study, the high percentage of extracolonic findings in $89 \%$ of the patients is most probably due to both examining a symptomatic high risk patient cohort and considering all findings without separately categorizing them as of low, moderate, or high importance. Furthermore, categorizing extracolonic findings may be quite difficult due to subjective rating. In our study, we detected a significantly higher number of extracolonic findings in patients with IV contrast-enhanced CT colonography compared to those with non-enhanced CT scans. On the other hand, the difference between these patients concerning further work up was found not to be statistically significant.

Only a few studies have explored the impact of extracolonic findings at CT colonography on further work up or therapy predominately in symptomatic patients [9-11]. In 
the present study, $25 \%$ of patients were found to have previously unknown extracolonic findings with an impact on further work up or therapy, a rate higher than the 2 to $13 \%$ of previous studies. Moreover, in our study 303 extracolonic findings were detected in 102 patients compared to 232 findings in 111 patients (Hellstrom et al.), 16 in 100 patients (Edwards et al.), and 68 in 49 patients (Ginnerup Pedersen et al.) in other studies [7, 9, 10]. Hara et al. obtained 151 extracolonic findings in a study population of 264 patients and detected a low rate compared to that of Hellstrom et al. and our own. A possible explanation for this discrepancy - besides the higher mean age and symptomatic high-risk nature of our patient population (66 years), which accord with previous studies - may be our use of IV contrast medium in the majority of our patients, which was not done in other studies and appears to account in large part for the high rate of extracolonic findings in our study. In addition, our study included abdominal CT scanning with standard doses, whereas both Hara et al. and Edwards et al. [10,11] employed a low dose CT protocol.

The majority of patients in the present study were given IV contrast medium, something that was not done in other studies on extracolonic findings. A higher number of all our extracolonic findings were obtained at IV contrastenhanced CT colonography. Although the present study was not designed to compare directly the conspicuity of benign and malignant lesions on unenhanced and IV-enhanced CT since patients initially received either unenhanced CT or IV contrast-enhanced CT and not both at a time, IV contrast-enhanced CT increased the conspicuity of certain lesions such as venous thrombosis of the external iliac vein, splenic vein, and portal vein, which probably could not have been diagnosed on non-enhanced CT. In addition, an inflamed aortic aneurysm with an aortoduodenal fistula was found (Fig. 5), which would have been almost impossible to detect without IV contrast enhancement. The use of IV contrast enhancement also enabled the readers to distinguish between solid and cystic lesions, especially in kidney and liver (Fig. 6). Other important findings, however, such as an aortic aneurysm, mass lesion, enlarged lymph nodes and a cholecystitis, would probably have been diagnosed even with non-enhanced CT scans. IV contrast medium has been shown to improve the detection of colorectal polyps and carcinomas and to facilitate the differentiation of solid lesions from residual colonic fluid and stool on axial images [6, 14-19]. It may also improve detection of colorectal polyps and help to reduce the need for additional imaging studies to clarify potential extracolonic findings, although in the present study the number of follow-up imaging studies was higher after contrast-enhanced CT scans due to the higher number of extracolonic findings.

The use of IV contrast in symptomatic patients combines CT colonography with a routine contrast-enhanced abdominal $\mathrm{CT}$ scan, resulting in a one stop diagnostic in- vestigation instead of needing additional imaging. However, the added risks and cost of administering IV contrast medium probably preclude its use as part of screening CT colonography protocols for large populations [20].

In the present study, axial images with a small slice thickness $(2 \mathrm{~mm})$ and an overlapping reconstruction interval of $1 \mathrm{~mm}$ were used to detect colorectal polyps; such images are not always used for viewing of abdominal CT in routine clinical practice. In addition, our analysis of extracolonic findings was facilitated by using both supine and prone CT datasets, a workstation with dynamic window settings, cine mode, and multiplanar reconstructions. All of these factors may have improved our detection rate of extracolonic lesions [9].

CT colonography requires scanning of the patient in both supine and prone positions, because a change in body position redistributes the intraluminal content, thus improving visualization of colorectal polyps [21]. Double scanning, however, means the patient receives a double dose of radiation, which is of particular importance in young patients and in screening programs. Hara et al. reduced the radiation dose by lowering the tube current on non-enhanced CT colonography and found the image quality to be sufficient for evaluation of the bowel wall due to the high contrast between intraluminal air and adjacent soft tissues [22]. However, a lowered tube current entails an increase in image noise, which may adversely affect detection of extracolonic findings, especially in solid abdominal organs. In the present study, CT colonography was performed with a standard abdominal $\mathrm{CT}$ radiation dose $(120 \mathrm{kV}, 200 \mathrm{mAs})$, which possibly contributed to the high rate of extracolonic findings, especially of smaller lesions, which would probably have eluded detection on noisy low dose images.

Extracolonic findings at $\mathrm{CT}$ colonography were assessed in three previous studies using a tube current of $70 \mathrm{mAs}$ [10-12]. The reported prevalence of extracolonic findings between 15 and $69 \%$ is lower than that reported by Hellstrom et al. (85\% of patients) at $125 \mathrm{mAs}$ and in our study ( $89 \%$ of patients) at $200 \mathrm{mAs}$. In our study, this discrepancy may also be related to the administration of IV contrast medium, differences in patient selection, and differences in definitions of extracolonic findings.

The frequency of extracolonic findings on CT colonography in a screening population was reported by Gluecker et al. to be $69 \%$ in 681 patients using unenhanced low-dose CT colonography [12]. Of these, only $1.3 \%$ resulted in subsequent work up or therapy, which is a lower number than the $25 \%$ in our study involving a symptomatic patient cohort. However, $10 \%$ of extracolonic findings in their study were considered to be clinically important, adding benefit to the screening intervention. Detection of incidental extracolonic findings, however, has many advantages, such as early detection of malignant disease or of an unruptured abdominal aortic aneurysm. Early treatment can improve a patient's prognosis and decrease costs owing 
to less complicated surgical procedures and shorter hospital stays. Clinically important incidental extracolonic findings leading to further work up were quite common in our study. Extracolonic findings, however, may also lead to unnecessary work up, causing unnecessary patient anxiety and entailing higher costs and superfluous exposure to radiation. They must therefore be considered a possible disadvantage of CT colonography for routine diagnostic work up and screening [11].

To summarize, in symptomatic patients IV contrastenhanced CT colonography produced more extracolonic findings, a greater proportion of which led to further work up and treatment, than did non-enhanced CT colonography, making IV contrast-enhanced CT colonography a one-stop diagnostic investigation. Future studies must determine whether the added risks and cost of IV contrast medium preclude its use as part of CT colonography screening protocols for large populations.

Acknowledgements This study was supported by a research grant from the Helmut-Horten-Foundation, Lugano, Switzerland. The authors wish to thank the CT technologists at the Institute of Radiology, Inselspital Bern, for their excellent expert assistance.

\section{References}

1. Vining DJ, Gelfand DW, Bechthold RE, Scharling ES, Grishaw EK, Shifrin RY (1994) Technical feasibility of colon imaging with helical CT and virtual reality. Am J Roentgenol 162:104

2. Yee J, Akerkar GA, Hung RK, Steinauer-Gebauer AM, Wall SD, McQuaid KR (2001) Colorectal neoplasia: performance characteristics of CT colonography for detection in 300 patients. Radiology 219:685-692

3. Pickhardt PJ, Choi R, Hwang I, Butler JA, Puckett ML, Hildebrandt HA, Wong RK, Nugent PA, Mysliwiec PA, Schindler WR (2003) Computed tomographic virtual colonoscopy to screen for colorectal neoplasia in asymptomatic adults. N Engl J Med 349:2191-2200

4. Bruzzi JF, Moss AC, Brennan DD, MacMathuna P, Fenlon HM (2003) Efficacy of IV Buscopan as a muscle relaxant in CT colonography. Eur Radiol 13:2264-2270

5. Taylor SA, Halligan S, Burling D, Morley S, Bassett P, Atkin W, Bartram CI (2004) CT colonography: effect of experience and training on reader performance. Eur Radiol 14:1025-1033

6. Hoppe H, Netzer P, Spreng A, Quattropani C, Mattich J, Dinkel H-P (2004) Prospective evaluation of contrast enhanced CT colonography and conventional colonoscopy for detection of colorectal neoplasms in a single institutional study using second-look colonoscopy with discrepant results. Am J Gastroenterol 99:1924-1935

7. Ginnerup Pedersen B, Rosenkilde M, Christiansen TEM, Laurberg S (2003) Extracolonic findings at computed tomography colonography are a challenge. Gut 52:1744-1747
8. Ng CS, Doyle TC, Courtney HM, Campbell GA, Freeman AH, Dixon AK (2004) Extracolonic findings in patients undergoing abdomino-pelvic CT for suspected colorectal carcinoma in the frail and disabled patient. Clin Radiol 59:421-430

9. Hellstrom M, Svensson E, Lasson A (2004) Extracolonic and incidental findings on CT colonography (virtual colonoscopy). Am J Roentgenol 182:631-638

10. Edwards JT, Wood CJ, Mendelson RM, Forbes GM (2004) Extracolonic findings at virtual colonoscopy: implications for screening programs. Am J Gastroenterol 96:3009-3012

11. Hara AK, Daniel Johnson C, MacCarty RL, Welch TJ (2000) Incidental extracolonic findings at CT colonography. Radiology 215:353-357

12. Gluecker TM, Johnson CD, Wilson LA, MacCarty RL, Welch TJ, Vanness DJ, Ahlquist DA (2003) Extracolonic findings at CT colonography: evaluation of prevalence and cost in a screening population. Gastroenterology 124:911-916

13. Panicek DM, Giess CS, Schwartz LH (1997) Qualitative assessment of liver for fatty infiltration on contrast-enhanced CT: is muscle a better standard of reference than spleen? J Comput Assist Tomogr 21:699-705

14. Morrin MM, Farrell RJ, Kruskal JB, Reynolds K, McGee JB, Raptopoulos V (2000) Utility of intravenously administered contrast material at CT colonography. Radiology 217:765-771

15. van Gelder RE, Venema HW, Serlie IW, Nio CY, Determann RM, Tipker CA, Vos FM, Glas AS, Bartelsman JF, Bossuyt PM, Lameris JS, Stoker J (2002) CT colonography at different radiation dose levels: feasibility of dose reduction. Radiology 224:25-33
16. Luboldt W, Mann C, Tryon CL, Vonthein R, Stueker D, Kroll M, Luz O, Claussen CD, Vogl TJ (2002) Computer-aided diagnosis in contrastenhanced CT colonography: an approach based on contrast. Eur Radiol 12:2236-2241

17. Hoppe H, Quattropani C, Spreng A, Mattich J, Netzer P, Dinkel H-P (2004) Virtual Colon Dissection with CT colonography compared to axial reading and conventional colonoscopy: preliminary results. Am J Roentgenol 182:1151-1158

18. Luboldt W, Kroll M, Wetter A, Toussaint TL, Hoepffner N, Holzer K, Kluge A, Vogl TJ (2004) Phase- and size-adjusted CT cut-off for differentiating neoplastic lesions from normal colon in contrast-enhanced CT colonography. Eur Radiol 14:2228-2235

19. Luboldt W, Tryon C, Kroll M, Toussaint TL, Holzer K, Hoepffner N, Vogl TJ (2005) Automated mass detection in contrast-enhanced CT colonography: an approach based on contrast and volume. Eur Radiol 15:247-253

20. Ferrucci JT (2001) Colon cancer screening with virtual colonoscopypromise, polyps, politics. Am J Roentgenol 177:975-988

21. Fletcher JG, Johnson CD, Welch TJ, MacCarty RL, Ahlquist DA, Reed JE, Harmsen WS, Wilson LA (2000) Optimization of CT colonography technique: prospective trial in 180 patients. Radiology 216:704-711

22. Hara AK, Johnson CD, Reed JE, Ahlquist DA, Nelson H, Ehman RL, Harmsen WS (1997) Reducing data size and radiation dose for CT colonography. Am J Roentgenol 168:11811184 